\title{
GAF: Generic Adaptation Framework
}

\author{
Evgeny Knutov* \\ Technische Universiteit Eindhoven \\ P.O. Box 513 5600MB \\ Eindhoven, The Netherlands \\ $+31-40-247-5154$ \\ e.knutov@tue.nl
}

\begin{abstract}
The Generic Adaptation Framework research project aims to develop a new reference model for the adaptive hypermedia research field. The new model will consider new developments, techniques and methodologies in the areas of adaptive hypermedia and adjacent fields.
\end{abstract}

\section{Keywords}

Adaptive hypermedia, reference model, generic framework.

\section{INTRODUCTION}

The research field of adaptive hypermedia and adaptive webbased information systems has been growing rapidly during the past ten years and this has resulted in new terms, concepts, models, prototypes and methodologies. The main existing reference model AHAM [1], developed in the beginning of this period, preceded many of these new developments. The "Generic Adaptation Framework" (or GAF) project aims at the research how these updated and new methodologies can fit into a new reference model of AHS, and provide a common reference in terms of both taxonomy and architecture.

\section{GENERIC ADAPTATION FRAMEWORK}

The adaptation process is usually defined by techniques and methods of AH used. Here we not only revisit the questions of adaptation process, methods and techniques, but also address issues of aligning all these question in a common structure, of a generic AHS.

Adaptation techniques and methods refer to means of providing adaptation and their generalization correspondingly and comprise the core of adaptation process. Every adaptation method shows a clear idea of the approach, but at the same time each method can be implemented by a number of different techniques, likewise some techniques may be used to implement several methods using the same knowledge representation. This set of techniques and methods comprises a toolkit of Adaptive Hypermedia [5]. Both techniques and methods can be applied to content, presentation and navigation adaptation. Here we distinguish adaptive presentation beyond the original content and navigation techniques (from [5]) and decide to differentiate the three forms of adaptation [4]: Content Adaptation Techniques, Adaptive
Presentation Techniques and Adaptive Navigation Techniques in a single diagram (Figure 1).

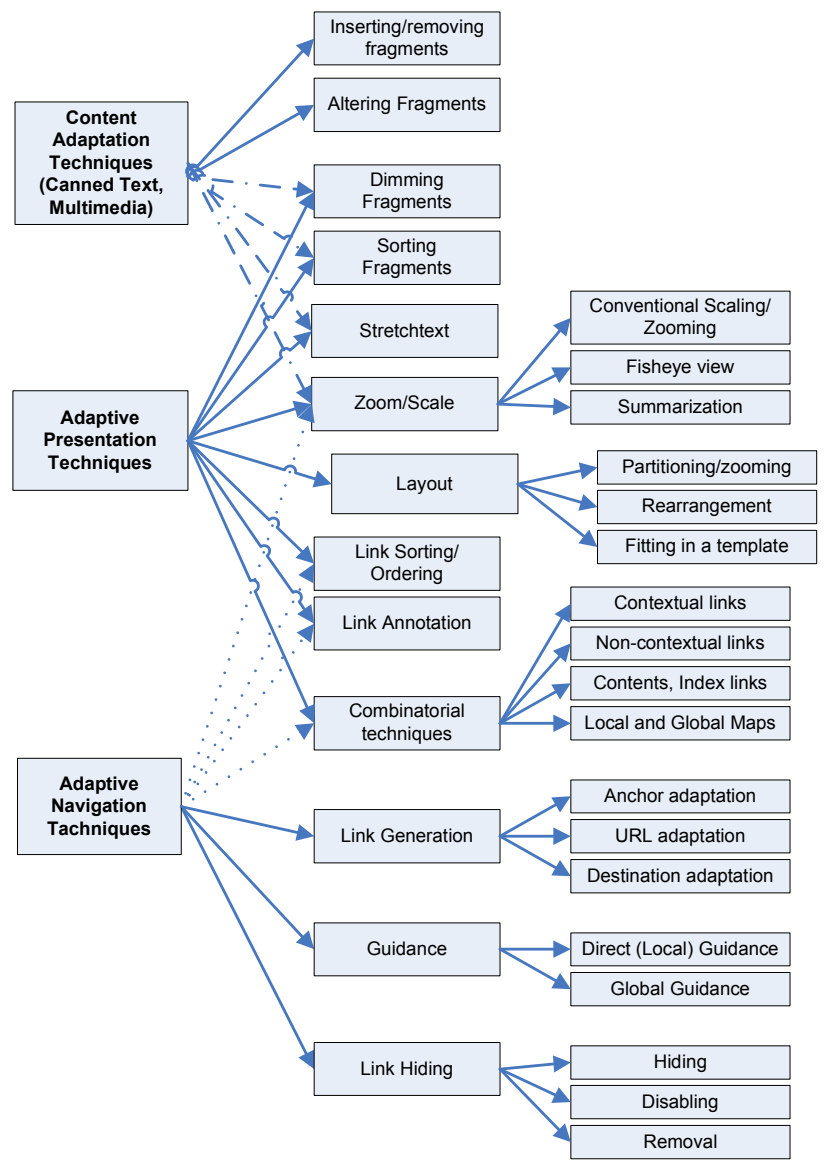

Figure 1. The New taxonomy of adaptation techniques

But not only methods and techniques define the reference model. Hereafter we present a draft architecture of a generic adaptation framework as a layered structure. Reference models started having layered architecture with the Dexter Model [6], later on Tower Model [7] became an extensible data model for Hyperdocuments intended to serve as the basis for integrating hypermedia systems with other information sources. Later the most well-known and important AHAM [1] was developed, followed by other models/systems, such as LAOS (where the aforementioned

\footnotetext{
${ }^{*}$ E. Knutov is a graduate student at the Eindhoven University of Technology, Department of Mathematics and Computer Science.
}

His academic advisor is Prof. Dr. P.M.E. De Bra. 
layered approach was elaborated), APeLS, the Munich model, Hera, etc. In our research we reconsider design issues for application independent generic AHS, review open questions of system extensibility introduced in adjacent research fields, and try to come up with the generic approach to build up an adaptive framework.

We also present a summary of new trends to compose a vision of future generic adaptive hypermedia systems (AHS). They are:

Ontologies: In order to provide interoperability adaptive applications must agree upon concept structures and meanings, therefore ontologies as a base concept structures are accepted in more and more research fields.

Open corpus adaptation: Even though open corpus is not a completely new research field, adaptive applications increasingly consider open corpus adaptation, where resources come from search results in large and dynamic learning object repositories or from a Web search engine.

Data Mining: These are valuable tools with respect to clustering users into groups based on their navigational patterns or capturing long term effects of adaptation rules and their automatic update.

Group-based adaptation: Most AHS perform adaptation to individual users, however we can extend this by taking group adaptation into account. Determining partitioning of the users into groups and applying this in the context of application model is yet another challenge.

Higher order adaptation: We see applications that not only monitor the user's behavior in order to perform adaptation, but also to adapt the adaptation behavior.

Context awareness: Context awareness (Context model) should allow systems and applications to be decoupled from the existing environment, and make them more sensitive to adapt in many other ways rather than a set of predefined rules.

Multimedia adaptation: a generic framework should provision a content type independence at any application level, providing a generalization of techniques and methods to work with.

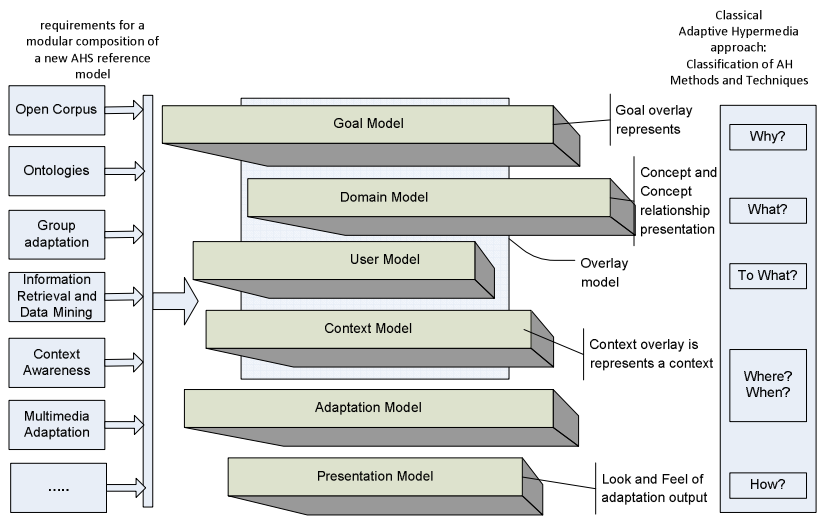

Figure 2. GAF conceptual scheme

Thus the main goal of GAF is to provide the generic architecture, to separate essential elements from optional and to define criteria to distinguish between these elements, to provide a modular structure that can be used either separately or together, depending on the needs of the intended application, that can be developed over generic framework to satisfy different needs. We have separated the layers in the conceptual scheme of the framework to match the original classification of $\mathrm{AH}$ methods and techniques, so that each layer is responsible for its own question (Why?, What?, To What?, Where?, When?, How? [5]). Therefore the generic model should facilitate the use of an arbitrary number of layers depending on the requirements of the application and contribute to the system extensibility.

\section{CONCLUSION AND FURTHER WORK}

The coming years will bring more use-cases of how AHS can provide adaptation, what techniques will be introduced, and what research areas will introduce new technologies in its evolution.

So far a study of existing methods was done to get a basic understanding of adaptive information systems., which has resulted in a survey paper providing an overview of an Adaptive Hypermedia field. A detailed comparison of major AHS systems was done to discover common parts and extract a generic modular structure of the systems. In this paper we presented a high level sketch of an architecture proposal, which brings us new challenges to investigate the applicability of different approaches used within different implementations and methodologies, as well as new developments in adaptive information systems (like open corpus adaptation, ontologies, group adaptation, data mining support, etc.), describe their interaction in the system and fit the overall GAF architectural proposals.

However, as a result of investigation even now we can foresee some further developments and research strategies of $\mathrm{AH}$ and thus try to come up with up-to-date requirements for a modular composition of a new AHS reference model.

\section{ACKNOWLEDGMENTS}

This research is supported by the NWO GAF Project.

\section{REFERENCES}

[1] De Bra, P., Houben, G.J., Wu, H., AHAM: A Dexter-based Reference Model for Adaptive Hypermedia, Proceedings of the ACM Conference on Hypertext and Hypermedia, pp. 147-156. (1999).

[2] De Bra, P., Aroyo, L., Chepegin, V., The Next Big Thing: Adaptive Web-Based Systems, Journal of Digital Information, vol. 5 issue 1 article 247. (2004).

[3] Aroyo, L., De Bra, P., Houben, G-J., Vdovjak, R., "Embedding information retrieval in adaptive hypermedia: IR meets AHA!', Hypermedia, 10(1), pp. 53-76, (2004).

[4] Knutov, E., De Bra, P., Pechenizkiy, M., AH 12 years later: a comprehensive survey of adaptive hypermedia methods and techniques, New Review of Hypermedia and Multimedia vol. 15, nr. 1, Taylor \& Francis, (2009). (to appear)

[5] Brusilovsky, P., "'Methods and techniques of adaptive hypermedia", User Modeling and User Adapted Interaction, 6(2_3), pp. 87-129, (1996).

[6] Halasz, F. and Schwartz, M., The Dexter Hypertext Reference Model. Communications of the ACM, 37(2), 3039, (1994)

[7] V.D. Aalst, W., De Bra, P., Houben, G.-J., Kornatzky, Y., Browsing Semantics in the "Tower" Model. Computer Science Notes, 93(47), 2-19, (1993) 
